\title{
Multilinear Singular Integrals and Commutators on Herz Space with Variable Exponent
}

\author{
Amjad Hussain ${ }^{1,2}$ and Guilian Gao ${ }^{1,3}$ \\ ${ }^{1}$ Department of Mathematics, Zhejiang University, Hangzhou 310027, China \\ ${ }^{2}$ Department of Mathematics, HITEC University, Taxila, Pakistan \\ ${ }^{3}$ School of Science, Hangzhou Dianzi University, Hangzhou 310018, China
}

Correspondence should be addressed to Amjad Hussain; ahabbasi123@yahoo.com

Received 7 October 2013; Accepted 23 December 2013; Published 12 February 2014

Academic Editors: V. Maiorov and Q. Xu

Copyright (C) 2014 A. Hussain and G. Gao. This is an open access article distributed under the Creative Commons Attribution License, which permits unrestricted use, distribution, and reproduction in any medium, provided the original work is properly cited.

The paper establishes some sufficient conditions for the boundedness of singular integral operators and their commutators from products of variable exponent Herz spaces to variable exponent Herz spaces.

\section{Introduction}

In recent years, the interest in multilinear analysis for studying boundedness properties of multilinear integral operators has grown rapidly. The subject was founded by Coifman and Meyer [1] in their seminal work on singular integral operators like Calderón commutators and pseudosdifferential operators having multiparameter function input. Subsequently, many authors including Christ and Journé [2], Kenig and Stein [3], and Grafakos and Torres [4] have substantially added to the exiting theory.

Let $K$ be a locally integrable function defined away from the diagonal $x=y_{1}=\cdots=y_{m}$ in $\left(\mathbb{R}^{n}\right)^{m+1}$, which for $C>0$ satisfies the estimates

$$
\left|K\left(x, y_{1}, \ldots, y_{m}\right)\right| \leq \frac{C}{\left(\left|x-y_{1}\right|+\cdots+\left|x-y_{m}\right|\right)^{m n}},
$$

and for $\epsilon>0$,

$$
\begin{aligned}
& \left|K\left(x, y_{1}, \ldots, y_{m}\right)-K\left(x^{\prime}, y_{1}, \ldots, y_{m}\right)\right| \\
& \quad \leq \frac{C\left|x-x^{\prime}\right|^{\epsilon}}{\left(\sum_{j=1}^{m}\left|x-y_{j}\right|\right)^{m n+\epsilon}},
\end{aligned}
$$

whenever $\left|x-x^{\prime}\right| \leq(1 / 2) \max \left\{\left|x-y_{1}\right|, \ldots,\left|x-y_{m}\right|\right\}$, and

$$
\begin{aligned}
& \left|K\left(x, y_{1}, \ldots, y_{i}, \ldots, y_{m}\right)-K\left(x^{\prime}, y_{1}, \ldots, y_{i}, \ldots, y_{m}\right)\right| \\
& \quad \leq \frac{C\left|y_{i}-y_{i}^{\prime}\right|^{\epsilon}}{\left(\sum_{j=1}^{m}\left|x-y_{j}\right|\right)^{m n+\epsilon}}
\end{aligned}
$$

whenever $\left|y_{i}-y_{i}^{\prime}\right| \leq(1 / 2) \max \left\{\left|x-y_{1}\right|, \ldots,\left|x-y_{m}\right|\right\}$ for all $1 \leq$ $i \leq m$. Then $K$ is called $m$-linear Calderón-Zygmund kernel. In this paper, we consider an $m$-linear singular integral operator $T$ associated with the kernel $K$, which is initially defined on product of the Schwartz space $\mathcal{S}\left(\mathbb{R}^{n}\right)$ and takes its values in the space of tempered distribution $\mathcal{S}^{\prime}\left(\mathbb{R}^{n}\right)$ such that

$$
\begin{aligned}
T & \left(f_{1}, \ldots, f_{m}\right)(x) \\
\quad & =\int_{\left(\mathbb{R}^{n}\right)^{m}} K\left(x, y_{1}, \ldots, y_{m}\right) f_{1}\left(y_{1}\right) \cdots f_{m}\left(y_{m}\right) d y_{1} \cdots d y_{m},
\end{aligned}
$$

for $x \notin \bigcap_{j=1}^{m} \operatorname{supp} f_{j}$, where $f_{1}, \ldots, f_{m} \in L_{C}^{\infty}\left(\mathbb{R}^{n}\right)$, the space of compactly supported bounded functions. If $T$ is bounded from $L^{p_{1}} \times \cdots \times L^{p_{m}}$ to $L^{p}$ with $1<p_{1} \cdots p_{m}<\infty$ and $1 / p_{1}+\cdots+1 / p_{m}=1 / p$, then we say that $T$ is an $m$-linear Calderón-Zygmund operator. It has been proved in [4] that 
$T$ is a bounded operator on product of Lebesgue spaces and endpoint weak estimates hold. For the boundedness of $T$ and its commutators on the product of Herz-type spaces we refer the reader to see $[5,6]$ and [7], respectively.

In the last few decades, however, a number of research papers have appeared in the literature which study the boundedness of integral operators, including the maximal function, singular operators, and fractional integral and commutators on function spaces with nonstandard growth conditions. Such kind of spaces is named as variable exponent function spaces which include variable exponent Lebesgue, Sobolev, Lorentz, Orlicz, and Herz-type function spaces. Among them the most fundamental and widely explored space is the Lebesgue space $L^{p(x)}$ with the exponent $p$ depending on the point $x$ of the space. We will describe it briefly in the next section; however, we refer to the book [8] and the survey paper [9] for historical background and recent developments in the theory of $L^{p(x)}$ spaces. Despite the progress made, the problems of boundedness of multilinear singular integral operators and their commutators on $L^{p(x)}$ spaces remain open. Recently, Huang and $\mathrm{Xu}$ [10] proved the boundedness of such integral operators on the product of variable exponent Lebesgue space. Motivated by their results, in this paper we will study the multilinear singular integral on Herz space with variable exponent. Similar results for the boundedness of commutators generated by these operators and BMO functions are also provided.

Herz-type spaces are an important class of function spaces in harmonic analysis. In [11, 12], Izuki independently introduced Herz space with variable exponent $p$, by keeping the remaining two exponents $\alpha$ and $q$ as constants. Variability of alpha was recently considered by Almeida and Drihem [13] in proving the boundedness results for some classical operators on such spaces. More recently, Samko [14] introduced the generalized Herz-type spaces where all the three exponents were allowed to vary. In this paper, we will study the multilinear singular operators on variable exponent Herz space $\dot{K}_{p(\cdot)}^{\alpha, q}$ introduced in [12].

Throughout this paper, $C$ denotes a positive constant which may change from one occurrence to another. The next section contains some basic definitions and the main results of this study. Finally, the last section includes the proofs of main results along with some supporting lemmas.

\section{Main Results}

Let $\Omega$ be a measurable subset of $\mathbb{R}^{n}$ with Lebesgue measure $|\Omega|>0$. Given a measurable function $p(\cdot): \Omega \rightarrow[1, \infty]$, then for some $\lambda>0$ we define the variable exponent Lebesgue space as

$$
L^{p(\cdot)}(\Omega):=\left\{f \text { is measurable }: \int_{\Omega}\left(\frac{|f(x)|}{\lambda}\right)^{p(x)} d x<\infty\right\}
$$

and the space $L_{\text {loc }}^{p(\cdot)}(\Omega)$ as

$$
L_{\text {loc }}^{p(\cdot)}(\Omega):=\left\{f: f \in L^{p(\cdot)}(K) \forall \text { compact subset } K \subset \Omega\right\} .
$$

The Lebesgue space $L^{p(\cdot)}(\Omega)$ becomes a Banach function space when equipped with the norm

$$
\|f\|_{L^{p(\cdot)}(\Omega)}:=\inf \left\{\lambda>0: \int_{\Omega}\left(\frac{|f(x)|}{\lambda}\right)^{p(x)} d x \leq 1\right\} .
$$

Given a locally integrable function $f$ on $\Omega$, the HardyLittlewood maximal operator $M$ is defined by

$$
M f(x):=\sup _{r>0} r^{-n} \int_{B(x, r) \cap \Omega}|f(y)| d y,
$$

where $B(x, r):=\left\{y \in \mathbb{R}^{n}:|x-y|<r\right\}$. We denote

$$
\begin{aligned}
& p_{-}:=\operatorname{essinf}\{p(x): x \in \Omega\}, \\
& p_{+}:=\operatorname{ess} \sup \{p(x): x \in \Omega\} .
\end{aligned}
$$

We also define

$$
\begin{aligned}
\mathscr{P}(\Omega):= & \left\{p(\cdot): p_{-}>1, p_{+}<\infty\right\}, \\
\mathscr{B}(\Omega):= & \{p(\cdot): p(\cdot) \in \mathscr{P}(\Omega), \\
& \left.M \text { is bounded on } L^{p(\cdot)}(\Omega)\right\} .
\end{aligned}
$$

Cruz-Uribe et al. [15] and Nekvinda [16] independently proved the following sufficient conditions for the boundedness of $M$ on $L^{p(\cdot)}(\Omega)$.

Proposition 1. Let $\Omega$ be an open set. If $p(\cdot) \in \mathscr{P}(\Omega)$ satisfies

$$
\begin{gathered}
|p(x)-p(y)| \leq \frac{C}{-\log (|x-y|)}, \quad \text { if }|x-y| \leq \frac{1}{2}, \\
|p(x)-p(y)| \leq \frac{C}{\log (e+|x|)}, \quad \text { if }|y| \geq|x|,
\end{gathered}
$$

then one has $p(\cdot) \in \mathscr{B}(\Omega)$.

Define $\mathscr{P}^{0}(\Omega)$ to be the set of measurable functions $p$ : $\Omega \rightarrow(0, \infty)$ such that

$$
\begin{gathered}
p_{-}:=\operatorname{essinf}\{p(x): x \in \Omega\}>0, \\
p_{+}:=\operatorname{ess} \sup \{p(x): x \in \Omega\}<\infty .
\end{gathered}
$$

Given $p \in \mathscr{P}^{0}(\Omega)$, one can define the space $L^{p(\cdot)}$ as above. Since we will not use it in the this paper, we omit the details here and refer the reader to see $[10,17]$.

Let $B_{k}:=\left\{x:|x| \leq 2^{k}\right\}, A_{k}=B_{k} \backslash B_{k-1}$ and $\chi_{k}=\chi_{A_{k}}$ be the characteristic function of the set $A_{k}$ for $k \in \mathbb{Z}$.

Definition 2. For $\alpha \in \mathbb{R}, 0<q \leq \infty$ and $p(\cdot) \in \mathscr{P}\left(\mathbb{R}^{n}\right)$, the homogeneous Herz space with variable exponent $K_{p(\cdot)}^{\alpha, q}\left(\mathbb{R}^{n}\right)$ is defined by

$$
\dot{K}_{p(\cdot)}^{\alpha, q}\left(\mathbb{R}^{n}\right):=\left\{L_{\text {loc }}^{p(\cdot)}\left(\mathbb{R}^{n} \backslash\{0\}\right):\|f\|_{\dot{K}_{p(\cdot)}^{\alpha, q}\left(\mathbb{R}^{n}\right)}<\infty\right\},
$$


where

$$
\|f\|_{\dot{K}_{p(\cdot)}^{\alpha, q}\left(\mathbb{R}^{n}\right)}:=\left\{\sum_{k=-\infty}^{\infty} 2^{k \alpha q}\left\|f \chi_{k}\right\|_{L^{p(\cdot)}\left(\mathbb{R}^{n}\right)}^{q}\right\}^{1 / q} .
$$

In the sequel, unless stated otherwise, we will work on the whole space $\mathbb{R}^{n}$ and will not mention it. Taking $b_{1}$ and $b_{2}$ in $B M O$, Huang and $\mathrm{Xu}$ in [10] define the three commutator operators for suitable functions $f$ and $g$. One of them is the operator

$$
\begin{aligned}
& {\left[b_{1}, b_{2}, T\right](f, g)(x)} \\
& =b_{1}(x) b_{2}(x) T(f, g)(x)-b_{1}(x) T\left(f, b_{2} g\right)(x) \\
& \quad-b_{2}(x) T\left(b_{1} f, g\right)(x)+T\left(b_{1} f, b_{2} g\right)(x) .
\end{aligned}
$$

As corollaries of their main results they give the following estimates.

Theorem A. Let $T$ be 2-linear Calderón-Zygmund operator and $p(\cdot) \in \mathscr{P}^{0}\left(\mathbb{R}^{n}\right)$. If $p_{1}(\cdot), p_{2}(\cdot) \in \mathscr{B}\left(\mathbb{R}^{n}\right)$ such that $1 / p(x)=$ $1 / p_{1}(x)+1 / p_{2}(x)$, then there exists a constant $C$ independent of the functions $f, f_{h} \in L^{p_{1}(\cdot)}, g, g_{h} \in L^{p_{2}(\cdot)}$ and $h \in \mathbb{N}$ such that

$$
\begin{gathered}
\|T(f, g)\|_{L^{p(\cdot)}} \leq C\|f\|_{L^{p_{1}(\cdot)}}\|g\|_{L^{p_{2}(\cdot)}}, \\
\left\|\left(\sum_{h=1}^{\infty}\left|T\left(f_{h}, g_{h}\right)\right|^{r}\right)^{1 / r}\right\|_{L^{p^{(\cdot)}}} \leq C\left\|\left(\sum_{h=1}^{\infty}\left|f_{h}\right|^{r_{1}}\right)^{1 / r_{1}}\right\|_{L^{p_{1}(\cdot)}} \\
\times\left\|\left(\sum_{h=1}^{\infty}\left|g_{h}\right|^{r_{2}}\right)^{1 / r_{2}}\right\|_{L^{p_{2}(\cdot)}}
\end{gathered}
$$

hold, where $1<r_{l}<\infty$ for $l=1,2$ and $1 / r=1 / r_{1}+1 / r_{2}$.

Theorem B. Let $T$ be 2-linear Calderón-Zygmund operator, $b_{1}, b_{2} \in B M O\left(\mathbb{R}^{n}\right)$, and $p(\cdot) \in \mathscr{P}^{0}\left(\mathbb{R}^{n}\right)$. If $p_{1}(\cdot), p_{2}(\cdot) \in \mathscr{B}\left(\mathbb{R}^{n}\right)$ such that $1 / p(x)=1 / p_{1}(x)+1 / p_{2}(x)$, then there exists a constant $C$ independent of the functions $f, f_{h} \in L^{p_{1}(\cdot)}, g, g_{h} \in$ $L^{p_{2}(\cdot)}$ and $h \in \mathbb{N}$ such that

$$
\begin{aligned}
& \left\|\left[b_{1}, b_{2}, T\right](f, g)\right\|_{L^{p(\cdot)}} \leq C\left\|b_{1}\right\|_{*}\left\|b_{2}\right\|_{*}\|f\|_{L^{p_{1}(\cdot)}}\|g\|_{L^{p^{2}(\cdot)}}, \\
& \left\|\left(\sum_{h=1}^{\infty}\left|\left[b_{1}, b_{2}, T\right]\left(f_{h}, g_{h}\right)\right|^{r}\right)^{1 / r}\right\|_{L^{p(\cdot)}} \\
& \leq\left\|\left(\sum_{h=1}^{\infty}\left|f_{h}\right|^{r_{1}}\right)^{1 / r_{1}}\right\|\left\|_{L^{p_{1}(\cdot)}}\right\|\left(\sum_{h=1}^{\infty}\left|g_{h}\right|^{r_{2}}\right)^{1 / r_{2}} \| \\
& \quad \times C\left\|b_{1}\right\|_{*}\left\|b_{2}\right\|_{*}
\end{aligned}
$$

hold, where $1<r_{l}<\infty$ for $l=1,2$ and $1 / r=1 / r_{1}+1 / r_{2}$.

Motivated by these results, here we give the following two theorems.

Theorem 3. Let $T$ be 2-linear Calderón-Zygmund operator and $p(\cdot) \in \mathscr{P}\left(\mathbb{R}^{n}\right)$. Furthermore, let $p_{1}(\cdot), p_{2}(\cdot) \in \mathscr{B}\left(\mathbb{R}^{n}\right)$, $0<q_{l}<\infty,-n \delta_{p_{l}}<\alpha_{l}<n \delta_{p_{l}^{\prime}}, l=1,2$, where $\delta_{p_{l}}, \delta_{p_{l}^{\prime}}>0$ are constants defined in the next section such that $1 / p(x)=$ $1 / p_{1}(x)+1 / p_{2}(x), 1 / q=1 / q_{1}+1 / q_{2}$, and $\alpha=\alpha_{1}+\alpha_{2}$. If $T$ is bounded from $L^{p_{1}(\cdot)} \times L^{p_{2}(\cdot)}$ to $L^{p(\cdot)}$, then

$$
\begin{gathered}
\|T(f, g)\|_{\dot{K}_{p(\cdot)}^{\alpha, q}}^{\alpha} \leq C\|f\|_{\dot{K}_{p_{1}(\cdot)}^{\alpha_{1}, q_{1}}}\|g\|_{\dot{K}_{p_{2}(\cdot)}^{\alpha_{2}, q_{2}}}, \\
\left\|\left(\sum_{h=1}^{\infty}\left|T\left(f_{h}, g_{h}\right)\right|^{r}\right)^{1 / r}\right\|_{\dot{K}_{p(\cdot)}^{\alpha, q}} \leq C\left\|\left(\sum_{h=1}^{\infty}\left|f_{h}\right|^{r_{1}}\right)^{1 / r_{1}}\right\| \|_{\dot{K}_{p_{1}(\cdot)}^{\alpha_{1}, q_{1}}} \\
\times\left\|\left(\sum_{h=1}^{\infty}\left|g_{h}\right|^{r_{2}}\right)^{1 / r_{2}}\right\| \|_{\dot{K}_{p_{2}(\cdot)}^{\alpha_{2}, q_{2}}}
\end{gathered}
$$

hold for all $f, f_{h} \in \dot{K}_{p_{1}(\cdot)}^{\alpha_{1}, q_{1}}, g, g_{h} \in \dot{K}_{p_{2}(\cdot)}^{\alpha_{2}, q_{2}}$, where $1<r_{l}<\infty$ for $l=1,2$ and $1 / r=1 / r_{1}+1 / r_{2}$.

Theorem 4. Let $T$ be 2-linear Calderón-Zygmund operator, $b_{1}, b_{2} \in B M O\left(\mathbb{R}^{n}\right)$, and $p(\cdot) \in \mathscr{P}\left(\mathbb{R}^{n}\right)$. Furthermore, let $p_{1}(\cdot), p_{2}(\cdot) \in \mathscr{B}\left(\mathbb{R}^{n}\right), 0<q_{l}<\infty,-n \delta_{p_{l}}<\alpha_{l}<n \delta_{p_{l}^{\prime}}, l=1,2$, where $\delta_{p_{l}}, \delta_{p_{l}^{\prime}}>0$ are constants defined in the next section, such that $1 / p(x)=1 / p_{1}(x)+1 / p_{2}(x), 1 / q=1 / q_{1}+1 / q_{2}$, $\alpha=\alpha_{1}+\alpha_{2}$. If $\left[b_{1}, b_{2}, T\right]$ is bounded from $L^{p_{1}(\cdot)} \times L^{p_{2}(\cdot)}$ to $L^{p^{(\cdot)}}$, then

$$
\begin{gathered}
\left\|\left[b_{1}, b_{2}, T\right](f, g)\right\|_{\dot{K}_{p(\cdot)}^{\alpha, q}} \leq C\left\|b_{1}\right\|_{*}\left\|b_{2}\right\|_{*}\|f\|_{\dot{K}_{p_{1}(\cdot)}^{\alpha_{1}, q_{1}}}\|g\|_{\dot{K}_{p_{2}(\cdot)}^{\alpha_{2}, q_{2}}}, \\
\left\|\left(\sum_{h=1}^{\infty}\left|\left[b_{1}, b_{2}, T\right]\left(f_{h}, g_{h}\right)\right|^{r}\right)^{1 / r}\right\|_{\dot{K}_{p(\cdot)}^{\alpha, q}}^{\alpha}\left\|\left(\sum_{h=1}^{\infty}\left|f_{h}\right|^{r_{1}}\right)^{1 / r_{1}}\right\|\left\|_{\dot{K}_{p_{1}(\cdot)}^{\alpha_{1}, q_{1}}}\right\|\left(\sum_{h=1}^{\infty}\left|g_{h}\right|^{r_{2}}\right)^{1 / r_{2}}\|\|_{\dot{K}_{p_{2}(\cdot)}^{\alpha_{2}, q_{2}}} \\
\quad \times C\left\|b_{1}\right\|_{*}\left\|b_{2}\right\|_{*}
\end{gathered}
$$

hold for all $f, f_{h} \in \dot{K}_{p_{1}(\cdot)}^{\alpha_{1}, q_{1}}, g, g_{h} \in \dot{K}_{p_{2}(\cdot)}^{\alpha_{2}, q_{2}}$, where $1<r_{l}<\infty$ for $l=1,2$ and $1 / r=1 / r_{1}+1 / r_{2}$.

\section{Proofs of the Main Results}

In this section, we will prove main results stated in the last section. The ideas of these proofs mainly come from $[5,7]$. We use the notation $p^{\prime}(x)$ to denote the conjugate index of $p(x)$. Here we give some lemmas which will be helpful in proving Theorems 3 and 4 .

Lemma 5 (see $[10,18]$, generalized Hölder's inequality). Let $p, p_{1}, p_{2} \in \mathscr{P}\left(\mathbb{R}^{n}\right)$.

(a) If $f \in L^{p(\cdot)}, g \in L^{p^{\prime}(\cdot)}$, then one has

$$
\|f g\|_{L^{1}} \leq C_{p}\|f\|_{L^{p(\cdot)}}\|g\|_{L^{p^{\prime}(\cdot)}},
$$

where $C_{p}=1+1 / p_{-}-1 / p_{+}$ 
(b) If $f \in L^{p_{1}(\cdot)}, g \in L^{p_{2}(\cdot)}$ and $1 / p(x)=1 / p_{1}(x)+$ $1 / p_{2}(x)$, then there exists a constant $C_{p, p_{1}}$ such that

$$
\|f g\|_{L^{p(\cdot)}} \leq C_{p, p_{1}}\|f\|_{L^{p_{1}(\cdot)}}\|g\|_{L^{p_{2}(\cdot)}}
$$

holds, where $C_{p, p_{1}}=\left(1+1 /\left(p_{1}\right)_{-}-1 /\left(p_{1}\right)_{+}\right)^{1 / p_{-}}$.

Lemma 6 (see [12]). If $p \in \mathscr{B}\left(\mathbb{R}^{n}\right)$, then there exist a constant $C>0$ such that for all balls $B$ in $\mathbb{R}^{n}$,

$$
\left\|\chi_{B}\right\|_{L^{(\cdot)}}\left\|\chi_{B}\right\|_{L^{\left.p^{\prime}()\right)}} \leq C|B| .
$$

Lemma 7 (see [12]). If $p \in \mathscr{B}\left(\mathbb{R}^{n}\right)$, then there exists constants $\delta, C>0$ such that for all balls $B$ in $\mathbb{R}^{n}$ and all measurable subsets $S \subset B$,

$$
\frac{\left\|\chi_{B}\right\|_{L^{p(\cdot)}}}{\left\|\chi_{S}\right\|_{L^{p^{(\cdot)}}}} \leq C \frac{|B|}{|S|}, \quad \frac{\left\|\chi_{S}\right\|_{L^{p^{(\cdot)}}}}{\left\|\chi_{B}\right\|_{L^{p(\cdot)}}} \leq C\left(\frac{|S|}{|B|}\right)^{\delta} .
$$

Lemma 8 (see $\left[19\right.$, Remark 1]). If $p_{l} \in \mathscr{B}\left(\mathbb{R}^{n}\right), l=1,2$, then by Proposition 1 one has $p_{l}^{\prime} \in \mathscr{B}\left(\mathbb{R}^{n}\right)$. Therefore, applying Lemma 7 , one can take positive constants $\delta_{p_{l}}, \delta_{p_{l}^{\prime}}>0$ such that

$$
\frac{\left\|\chi_{S}\right\|_{L^{p_{l}(\cdot)}}}{\left\|\chi_{B}\right\|_{L^{\left.p^{(} \cdot\right)}}} \leq C\left(\frac{|S|}{|B|}\right)^{\delta_{p_{l}}}, \quad \frac{\left\|\chi_{S}\right\|_{L^{p_{l}^{\prime}(\cdot)}}}{\left\|\chi_{B}\right\|_{L^{p_{l}^{\prime} \cdot()}}} \leq C\left(\frac{|S|}{|B|}\right)^{\delta_{p_{l}^{\prime}}}
$$

for all balls $B$ in $\mathbb{R}^{n}$ and all measurable subsets $S \subset B$.

Recently, Izuki [19] established a relationship between Lebesgue space with variable exponent and BMO space which can be stated in the form of the following lemma.

Lemma 9. One has that for all $b \in B M O\left(\mathbb{R}^{n}\right)$ and all $i, j \in \mathbb{Z}$ with $j>i$,

$$
\begin{aligned}
C^{-1}\|b\|_{*} & \leq \sup _{B: b a l l} \frac{1}{\left\|\chi_{B}\right\|_{L^{p(\cdot)}}}\left\|\left(b-b_{B}\right) \chi_{B}\right\|_{L^{p(\cdot)}} \leq C\|b\|_{*} \\
& \left\|\left(b-b_{B_{i}}\right) \chi_{B_{j}}\right\|_{L^{p(\cdot)}} \leq C(j-i)\|b\|_{*}\left\|\chi_{B_{j}}\right\|_{L^{p(\cdot)}} .
\end{aligned}
$$

The next lemma is the generalized Minkowski's inequality and is useful in proving vector valued inequalities.

Lemma 10 (see [19]). If $1<r<\infty$, then there exists a constant $C>0$ such that for all sequences of functions $\left\{f_{h}\right\}_{h=1}^{\infty}$ satisfying \|\|$\left\{f_{h}\right\}_{h}\left\|_{\ell^{r}}\right\|_{L^{1} \mathbb{R}^{n}}<\infty$,

$$
\left\{\sum_{h=1}^{\infty}\left(\int_{\mathbb{R}^{n}}\left|f_{h}(y)\right| d y\right)^{r}\right\}^{1 / r} \leq C \int_{\mathbb{R}^{n}}\left\{\sum_{h=1}^{\infty}\left|f_{h}(y)\right|^{r}\right\}^{1 / r} d y .
$$

Proof of Theorem 3. In order to make computations easy, first we have to prove the following inequality:

$$
\begin{aligned}
2^{k \alpha}\left\|T\left(f \chi_{i}, g \chi_{j}\right) \chi_{k}\right\|_{L^{p(\cdot)}} \leq & C D_{1}(k, i) 2^{i \alpha_{1}}\left\|f \chi_{i}\right\|_{L^{p_{1}(\cdot)}} \\
& \times D_{2}(k, j) 2^{j \alpha_{2}}\left\|g \chi_{j}\right\|_{L^{p_{2}(\cdot)}},
\end{aligned}
$$

where for $k, i \in \mathbb{Z}, l=1,2$,

$$
D_{l}(k, i)= \begin{cases}2^{(k-i)\left(\alpha_{l}-n \delta_{p_{l}^{\prime}}\right)}, & \text { if } i \leq k-2, \\ 1, & \text { if } k-1 \leq i \leq k+1 \\ 2^{(k-i)\left(\alpha_{l}+n \delta_{p_{l}}\right)}, & \text { if } i \geq k+2\end{cases}
$$

If $k-1 \leq i \leq k+1, k-1 \leq j \leq k+1$, then we have $2^{k} \sim 2^{i} \sim 2^{j}$; hence by the $L^{p(\cdot)}$ boundedness of $T$, we obtain

$$
\begin{aligned}
2^{k \alpha}\left\|T\left(f \chi_{i}, g \chi_{j}\right) \chi_{k}\right\|_{L^{p(\cdot)}} \leq & C 2^{i \alpha_{1}}\left\|f \chi_{i}\right\|_{L^{p^{1}(\cdot)}} 2^{j \alpha_{2}} \\
& \times\left\|g \chi_{j}\right\|_{L^{p_{2}(\cdot)}} \cdot
\end{aligned}
$$

In the other cases, we see that $\left|x-y_{1}\right|+\left|x-y_{2}\right| \sim 2^{\max (k, i, j)}$, for $x \in A_{k}, y_{1} \in A_{i}, y_{2} \in A_{j}$. Thus by the generalized Hölder's inequality,

$$
\begin{aligned}
\left|T\left(f \chi_{i}, g \chi_{j}\right)(x) \chi_{k}(x)\right| \leq & C 2^{-2 \max (k, i, j) n}\left\|f \chi_{i}\right\|_{1}\left\|g \chi_{j}\right\|_{1} \cdot \chi_{k}(x) \\
\leq & C 2^{-2 \max (k, i, j) n}\left\|f \chi_{i}\right\|_{L^{p_{1}(\cdot)}}\left\|\chi_{i}\right\|_{L^{p_{1}^{\prime}(\cdot)}} \\
& \times\left\|g \chi_{j}\right\|_{L^{p_{2}(\cdot)}}\left\|\chi_{j}\right\|_{L^{p_{2}^{\prime}(\cdot)}} \cdot \chi_{k}(x) .
\end{aligned}
$$

Applying Lemma 5, we have

$$
\begin{aligned}
& \left\|T\left(f \chi_{i}, g \chi_{j}\right) \chi_{k}\right\|_{L^{p(\cdot)}} \\
& \leq C 2^{-2 \max (k, i, j) n}\left\|f \chi_{i}\right\|_{L^{p^{1}(\cdot)}}\left\|\chi_{i}\right\|_{L^{p_{1}^{\prime}(\cdot)}} \\
& \quad \times\left\|g \chi_{j}\right\|_{L^{p_{2}(\cdot)}}\left\|\chi_{j}\right\|_{L^{p_{2}^{(\cdot)}}}\left\|\chi_{k}\right\|_{L^{p^{(\cdot)}}} \\
& \leq C 2^{-\max (k, i) n}\left\|f \chi_{i}\right\|_{L^{p_{1}(\cdot)}}\left\|\chi_{B_{i}}\right\|_{L^{p_{1}^{\prime}(\cdot)}}\left\|\chi_{B_{k}}\right\|_{L^{p_{1}(\cdot)}} \\
& \quad \times 2^{-\max (k, j) n}\left\|g \chi_{j}\right\|_{L^{p_{2}(\cdot)}}\left\|\chi_{B_{j}}\right\|_{L^{p_{2}^{\prime}(\cdot)}}\left\|\chi_{B_{k}}\right\|_{L^{p_{2}(\cdot)}} \cdot
\end{aligned}
$$

Now, for $i \leq k-2, j \leq k-2$, by Lemmas 6 and 8 , it is easy to show that

$$
\begin{aligned}
& \left\|T\left(f \chi_{i}, g \chi_{j}\right) \chi_{k}\right\|_{L^{p(\cdot)}}
\end{aligned}
$$

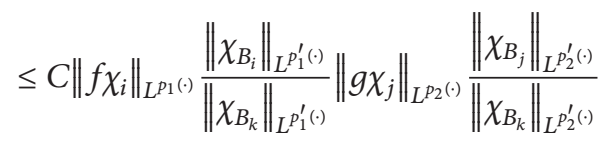

$$
\begin{aligned}
& \leq C\left\|f \chi_{i}\right\|_{L^{p_{1}(\cdot)}} 2^{-(k-i) n \delta_{p_{1}^{\prime}}}\left\|g \chi_{j}\right\|_{L^{p_{2}(\cdot)}} 2^{-(k-j) n \delta_{p_{2}^{\prime}}} \text {. }
\end{aligned}
$$

By a similar argument for $i \geq k+2, j \geq k+2$, we get

$$
\begin{aligned}
& \left\|T\left(f \chi_{i}, g \chi_{j}\right) \chi_{k}\right\|_{L^{p(\cdot)}}
\end{aligned}
$$

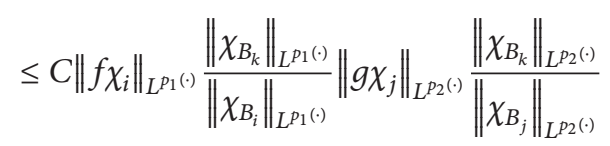

$$
\begin{aligned}
& \leq C\left\|f \chi_{i}\right\|_{L^{p_{1}(\cdot)}} 2^{(k-i) n \delta_{p_{1}}}\left\|g \chi_{j}\right\|_{L^{p_{2}(\cdot)}} 2^{(k-j) n \delta_{p_{2}}} .
\end{aligned}
$$


In view of (29)-(33), (27) is obvious. Now by Minkowski's inequality and (27), we get

$$
\begin{aligned}
2^{k \alpha}\left\|T(f, g) \chi_{k}\right\|_{L^{p()}} \leq & 2^{k \alpha}\left\|\sum_{i=-\infty}^{\infty} \sum_{j=-\infty}^{\infty} T\left(f \chi_{i}, g \chi_{j}\right) \chi_{k}\right\|_{L^{p(\cdot)}} \\
\leq & \sum_{i=-\infty}^{\infty} \sum_{j=-\infty}^{\infty} 2^{k \alpha}\left\|T\left(f \chi_{i}, g \chi_{j}\right) \chi_{k}\right\|_{L^{p(\cdot)}} \\
\leq & C \sum_{i=-\infty}^{\infty} D_{1}(k, i) 2^{i \alpha_{1}}\left\|f \chi_{i}\right\|_{L^{p_{1}(\cdot)}} \\
& \times \sum_{j=-\infty}^{\infty} D_{2}(k, j) 2^{j \alpha_{2}}\left\|g \chi_{j}\right\|_{L^{p_{2}(\cdot)}} .
\end{aligned}
$$

Since $1 / q=1 / q_{1}+1 / q_{2}$, then by definition

$$
\begin{aligned}
\| T & (f, g) \|_{\dot{K}_{p(\cdot)}^{\alpha, q}} \\
= & \left\{\sum_{k=-\infty}^{\infty} 2^{k \alpha q}\left\|T(f, g) \chi_{k}\right\|_{L^{p(\cdot)}}^{q}\right\}^{1 / q} \\
\leq & \left\{\sum _ { k = - \infty } ^ { \infty } \left(\sum_{i=-\infty}^{\infty} D_{1}(k, i) 2^{i \alpha_{1}}\left\|f \chi_{i}\right\|_{L^{p_{1}(\cdot)}}\right.\right. \\
\leq & C\left\{\sum_{k=-\infty}^{\infty}\left(\sum_{i=-\infty}^{\infty} D_{1}(k, j) 2^{j \alpha_{2}}\left\|g \chi_{j}\right\|_{L^{p_{2}(\cdot)}}\right)^{q}\right\}^{1 / q} \\
& \left.\times\left\{\sum_{k=-\infty}^{\infty}\left(\sum_{j=-\infty}^{\infty} D_{2}(k, j) 2^{j \alpha_{2}}\left\|g \chi_{j}\right\|_{L^{p_{2}(\cdot)}}\right)^{\alpha_{1}}\left\|f \chi_{i}\right\|_{L^{p_{1}(\cdot)}}\right)^{q_{1}}\right\}^{1 / q_{1}} \\
& =I_{1} \times I_{2} .
\end{aligned}
$$

It remains to show that $I_{1} \leq C\|f\|_{K_{\dot{K}_{1}(\cdot)}^{\alpha_{1}, q_{1}}}$ and $I_{2} \leq$ $C\|g\|_{\dot{K}_{p_{2}(-)}^{\alpha_{2}, q_{2}}}$. By symmetry, we only approximate $I_{1}$. If $0<q_{1}<$ 1 , then by the well-known inequality $\left(\sum\left|a_{i}\right|\right)^{q_{1}} \leq \sum\left|a_{i}\right|^{q_{1}}$ and the inequality

$$
\sum_{i=-\infty}^{\infty} D_{1}(k, i)^{\gamma}+\sum_{j=-\infty}^{\infty} D_{2}(k, j)^{\gamma}<\infty, \quad \text { for any } \gamma>0,
$$

we have

$$
\begin{aligned}
I_{1} & \leq C\left\{\sum_{i=-\infty}^{\infty} 2^{i \alpha_{1} q_{1}}\left\|f \chi_{i}\right\|_{L^{p_{1}(\cdot)}}^{q_{1}} \sum_{k=-\infty}^{\infty} D_{1}(k, i)^{q_{1}}\right\}^{1 / q_{1}} \\
& \leq C\left\{\sum_{i=-\infty}^{\infty} 2^{i \alpha_{1} q_{1}}\left\|f \chi_{i}\right\|_{L^{p_{1}(\cdot)}}^{q_{1}}\right\}^{1 / q_{1}}=C\|f\|_{\dot{K}_{p_{1}(\cdot)}^{\alpha_{1}, q_{1}}} .
\end{aligned}
$$

If $q_{1}>1$, Hölder's inequality and inequality (36) yield

$$
\begin{aligned}
I_{1} \leq C & \left\{\sum_{k=-\infty}^{\infty} \sum_{i=-\infty}^{\infty} D_{1}(k, i)^{q_{1} / 2} 2^{i \alpha_{1} q_{1}}\left\|f \chi_{i}\right\|_{L^{p_{1}(\cdot)}}^{q_{1}}\right. \\
& \left.\times\left\{\sum_{i=-\infty}^{\infty} D_{1}(k, i)^{q_{1}^{\prime} / 2}\right\}^{q_{1} / q_{1}^{\prime}}\right\}^{1 / q_{1}} \\
\leq & C\left\{\sum_{i=-\infty}^{\infty} 2^{i \alpha_{1} q_{1}}\left\|f \chi_{i}\right\|_{L^{p_{1}} \cdot(\cdot)}^{q_{1}} \sum_{k=-\infty}^{\infty} D_{1}(k, i)^{q_{1} / 2}\right\}^{1 / q_{1}} \\
\leq & C\left\{\sum_{i=-\infty}^{\infty} 2^{i \alpha_{1} q_{1}}\left\|f \chi_{i}\right\|_{L^{p_{1}} \cdot(\cdot)}^{q_{1}}\right\}^{1 / q_{1}}=C\|f\|_{\dot{K}_{p_{1}(\cdot)}^{\alpha_{1}, q_{1}} .}
\end{aligned}
$$

Therefore, for $0<q_{1}<\infty$

$$
I_{1} \leq C\|f\|_{\dot{K}_{\left.p_{1}()\right)}^{\alpha_{1}, q_{1}}}
$$

By symmetry, for $0<q_{2}<\infty$ we have

$$
I_{2} \leq C\|g\|_{\dot{K}_{p_{2} \cdot(\cdot)}^{\alpha_{2}, q_{2}}} \text {. }
$$

Finally, we obtain

$$
\|T(f, g)\|_{\dot{K}_{p(\cdot)}^{\alpha, q}} \leq C\|f\|_{\dot{K}_{p_{1}(\cdot)}^{\alpha_{1}, q_{1}}}\|g\|_{\dot{K}_{p_{2}(\cdot)}^{\alpha_{2}, q_{2}}} .
$$

By virtue of Lemma 10 and the fact that $1 / r=1 / r_{1}+1 / r_{2}$, it is easy to show that

$$
\begin{aligned}
\left\|\left(\sum_{h=1}^{\infty}\left|T\left(f_{h}, g_{h}\right)\right|^{r}\right)^{1 / r}\right\|_{\dot{K}_{p(\cdot)}^{\alpha, q}} \leq & C\left\|\left(\sum_{h=1}^{\infty}\left|f_{h}\right|^{r_{1}}\right)^{1 / r_{1}}\right\|_{\dot{K}_{\dot{p}_{1}(\cdot)}^{\alpha_{1}, q_{1}}} \\
& \times\left\|\left(\sum_{h=1}^{\infty}\left|g_{h}\right|^{r_{2}}\right)^{1 / r_{2}}\right\|_{\dot{K}_{p_{2}(\cdot)}^{\alpha_{2}, q_{2}}}
\end{aligned}
$$

holds for all $f_{h} \in \dot{K}_{p_{1}(\cdot)}^{\alpha_{1}, q_{1}}, g_{h} \in \dot{K}_{p_{2}(\cdot)}^{\alpha_{2}, q_{2}}$, where $1<r_{l}<\infty$ for $l=1,2$.

Thus the proof of Theorem 3 is complete.

Proof of Theorem 4. Similar to the proof of Theorem 3, for the case $k-1 \leq i \leq k+1, k-1 \leq j \leq k+1$, we use $L^{p(\cdot)}$ boundedness of $\left[b_{1}, b_{2}, T\right]$ to obtain

$$
\begin{aligned}
& 2^{k \alpha}\left\|\left[b_{1}, b_{2}, T\right]\left(f \chi_{i}, g \chi_{j}\right) \chi_{k}\right\|_{L^{p(\cdot)}} \\
& \quad \leq C\left\|b_{1}\right\|_{*}\left\|b_{2}\right\|_{*} 2^{i \alpha_{1}}\left\|f \chi_{i}\right\|_{L^{p_{1}(\cdot)}} 2^{j \alpha_{2}}\left\|g \chi_{j}\right\|_{L^{p_{2}(\cdot)}} .
\end{aligned}
$$

For other possibilities we have $\left|x-y_{1}\right|+\left|x-y_{2}\right| \sim 2^{\max (k, i, j)}$ for $x \in A_{k}, y_{1} \in A_{i}, y_{2} \in A_{j}$. Thus, we consider the following two cases.

Case $I(i \leq k-2, j \leq k-2)$. We denote $\left(b_{l}\right)_{B_{i}}$ by $b_{l i}$, where

$$
\left(b_{l}\right)_{B_{i}}=\frac{1}{\left|B_{i}\right|} \int_{B_{i}} b_{l}(x) d x, \quad l=1,2
$$


6

ISRN Mathematical Analysis

and consider the following decomposition:

$$
\begin{aligned}
& {\left[b_{1}, b_{2}, T\right]\left(f \chi_{i}, g \chi_{j}\right)(x) } \\
&=\left(b_{1}(x)-b_{1 i}\right)\left(b_{2}(x)-b_{2 j}\right) T\left(f \chi_{i}, g \chi_{j}\right)(x) \\
&-\left(b_{1}(x)-b_{1 i}\right) T\left(f \chi_{i},\left(b_{2}(\cdot)-b_{2 j}\right) g \chi_{j}\right)(x) \\
&-\left(b_{2}(x)-b_{2 j}\right) T\left(\left(b_{1}(\cdot)-b_{1 i}\right) f \chi_{i}, g \chi_{j}\right)(x) \\
&+T\left(\left(b_{1}(\cdot)-b_{1 i}\right) f \chi_{i},\left(b_{2}(\cdot)-b_{2 j}\right) g \chi_{j}\right)(x) \\
&= L_{1}(x)+L_{2}(x)+L_{3}(x)+L_{4}(x) .
\end{aligned}
$$

Thus,

$$
\left\|\left[b_{1}, b_{2}, T\right]\left(f \chi_{i}, g \chi_{j}\right) \chi_{k}\right\|_{L^{p(\cdot)}} \leq \sum_{m=1}^{4}\left\|\left(L_{m}\right) \chi_{k}\right\|_{L^{p(\cdot)}}=\sum_{m=1}^{4} J_{m} .
$$

Now, we will estimate each $J_{m}(m=1, \ldots, 4)$, separately. Applying Lemma 5, we have

$$
\begin{aligned}
\left|L_{1}(x)\right| \leq & C 2^{-2 k n}\left|b_{1}(x)-b_{1 i}\right|\left|b_{2}(x)-b_{2 j}\right| \\
& \times\left\|f \chi_{i}\right\|_{L^{1}}\left\|g \chi_{j}\right\|_{L^{1}} \\
\leq & C 2^{-2 k n}\left\|f \chi_{i}\right\|_{L^{p_{1}(\cdot)}}\left\|\chi_{i}\right\|_{L^{p_{1}^{\prime}(\cdot)}}\left\|g \chi_{j}\right\|_{L^{p_{2}(\cdot)}}\left\|\chi_{j}\right\|_{L^{p_{2}^{\prime}(\cdot)}} \\
& \times\left|b_{1}(x)-b_{1 i}\right|\left|b_{2}(x)-b_{2 j}\right| .
\end{aligned}
$$

Therefore, by virtue of generalized Hölder's inequality and Lemmas 6,8 , and 9 , we get

$$
\begin{aligned}
& J_{1}=\left\|\left(L_{1}\right) \chi_{k}\right\|_{L^{p())}} \\
& \leq C 2^{-2 k n}\left\|f \chi_{i}\right\|_{L^{p_{1}(\cdot)}}\left\|\chi_{i}\right\|_{L^{p_{1}^{\prime}(\cdot)}}\left\|g \chi_{j}\right\|_{L^{p^{2}(\cdot)}}\left\|\chi_{j}\right\|_{L^{p_{2}^{\prime}(\cdot)}} \\
& \times\left\|\left(b_{1}(x)-b_{1 i}\right)\left(b_{2}(x)-b_{2 j}\right) \chi_{k}\right\|_{L^{p(\cdot)}} \\
& \leq C 2^{-2 k n}\left\|f \chi_{i}\right\|_{\left.L^{p_{1}} \cdot\right)}\left\|\chi_{B_{i}}\right\|_{L^{p_{1}^{\prime} \cdot()}} \\
& \times\left\|\left(b_{1}(x)-b_{1 i}\right) \chi_{B_{k}}\right\|_{L^{p_{1}(\cdot)}}\left\|g \chi_{j}\right\|_{L^{p_{2}(\cdot)}}\left\|\chi_{B_{j}}\right\|_{L^{p_{2}^{\prime}(\cdot)}} \\
& \times\left\|\left(b_{2}(x)-b_{2 j}\right) \chi_{B_{k}}\right\|_{L^{p_{2}}(\cdot)} \\
& \leq C\left\|b_{1}\right\|_{*}\left\|b_{2}\right\|_{*} 2^{-2 k n}(k-i)\left\|f \chi_{i}\right\|_{L^{p_{1}(\cdot)}} \\
& \times\left\|\chi_{B_{i}}\right\|_{L^{p_{1}^{\prime}(\cdot)}}\left\|\chi_{B_{k}}\right\|_{L^{p_{1}(\cdot)}} \\
& \times(k-j)\left\|g \chi_{j}\right\|_{L^{p_{2}(\cdot)}}\left\|\chi_{B_{j}}\right\|_{L^{p_{2}^{\prime}(\cdot)}}\left\|\chi_{B_{k}}\right\|_{L^{p_{2}(\cdot)}} \\
& \leq C\left\|b_{1}\right\|_{*}\left\|b_{2}\right\|_{*}(k-i)\left\|f \chi_{i}\right\|_{L^{p_{1}(\cdot)}}
\end{aligned}
$$

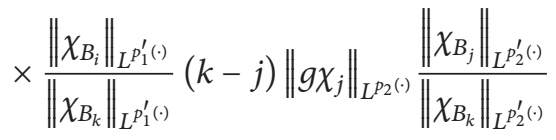

$$
\begin{aligned}
& \leq C\left\|b_{1}\right\|_{*}\left\|b_{2}\right\|_{*}\left\|f \chi_{i}\right\|_{L^{p_{1}(\cdot)}}(k-i) 2^{-(k-i) n \delta_{p_{1}^{\prime}}} \\
& \times\left\|g \chi_{j}\right\|_{L^{p_{2}(\cdot)}}(k-j) 2^{-(k-j) n \delta_{p_{2}^{\prime}}} .
\end{aligned}
$$

Similarly, using Lemma 9, we approximate $L_{2}$ as

$$
\begin{aligned}
\left|L_{2}\right| \leq & C 2^{-2 k n}\left|b_{1}(x)-b_{1 i}\right|\left\|f \chi_{i}\right\|_{L^{1}}\left\|\left(b_{2}(\cdot)-b_{2 j}\right) g \chi_{j}\right\|_{L^{1}} \\
\leq & C 2^{-2 k n}\left|b_{1}(x)-b_{1 i}\right|\left\|f \chi_{i}\right\|_{L^{p_{1}(\cdot)}}\left\|\chi_{i_{i}}\right\|_{L^{p_{1}^{\prime}(\cdot)}} \\
& \times\left\|g \chi_{j}\right\|_{L^{p_{2}(\cdot)}}\left\|\left(b_{2}(\cdot)-b_{2 j}\right) \chi_{j}\right\|_{L^{p_{2}^{\prime}(\cdot)}} \\
\leq & C\left\|b_{2}\right\|_{*} 2^{-2 k n}\left|b_{1}(x)-b_{1 i}\right|\left\|f \chi_{i}\right\|_{L^{p_{1}(\cdot)}} \\
& \times\left\|\chi_{B_{i}}\right\|_{L^{p_{1}^{\prime}(\cdot)}}\left\|g \chi_{j}\right\|_{L^{p_{2}(\cdot)}}\left\|\chi_{B_{j}}\right\|_{L_{2}^{p_{2}^{\prime}(\cdot)}} .
\end{aligned}
$$

Therefore, in view of Lemmas 6, 9, and 8, we have

$$
\begin{aligned}
& J_{2}=\left\|\left(L_{2}\right) \chi_{k}\right\|_{L^{p())}} \\
& \leq C\left\|b_{2}\right\|_{*} 2^{-2 k n}\left\|f \chi_{i}\right\|_{L^{p_{1}(\cdot)}}\left\|\chi_{B_{i}}\right\|_{L^{\left.p_{1}^{(} \cdot\right)}} \\
& \times\left\|\left(b_{1}(x)-b_{1 i}\right) \chi_{k}\right\|_{L^{p^{(\cdot)}}}\left\|g \chi_{j}\right\|_{L^{p^{2}(\cdot)}}\left\|\chi_{B_{j}}\right\|_{L^{p_{2}^{\prime}(\cdot)}} \\
& \leq C\left\|b_{1}\right\|_{*}\left\|b_{2}\right\|_{*} 2^{-2 k n}\left\|f \chi_{i}\right\|_{L^{p_{1}(\cdot)}}\left\|\chi_{B_{i}}\right\|_{L^{p_{1}^{\prime}(\cdot)}} \\
& \times(k-i)\left\|\chi_{B_{k}}\right\|_{L^{p(\cdot)}}\left\|g \chi_{j}\right\|_{L^{p_{2}(\cdot)}}\left\|\chi_{B_{j}}\right\|_{L^{p^{p^{\prime}}(\cdot)}} \\
& \leq C\left\|b_{1}\right\|_{*}\left\|b_{2}\right\|_{*}(k-i)\left\|f \chi_{i}\right\|_{L^{p_{1}(\cdot)}}
\end{aligned}
$$

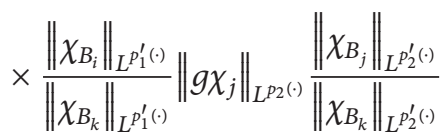

$$
\begin{aligned}
& \leq C\left\|b_{1}\right\|_{*}\left\|b_{2}\right\|_{*}\left\|f \chi_{i}\right\|_{L^{p_{1}(\cdot)}}(k-i) 2^{-(k-i) n \delta_{p_{1}^{\prime}}} \\
& \times\left\|g \chi_{j}\right\|_{L^{p_{2}(\cdot)}} 2^{-(k-j) n \delta_{p_{2}^{\prime}}} .
\end{aligned}
$$

By symmetry, the estimate for $J_{3}$ is similar to that for $J_{2}$; therefore,

$$
\begin{aligned}
J_{3} \leq & C\left\|b_{1}\right\|_{*}\left\|b_{2}\right\|_{*}\left\|f \chi_{i}\right\|_{L^{p_{1}(\cdot)}} 2^{-(k-i) n \delta_{p_{1}^{\prime}}} \\
& \times\left\|g \chi_{j}\right\|_{L^{p_{2}(\cdot)}}(k-j) 2^{-(k-j) n \delta_{p_{2}^{\prime}}} .
\end{aligned}
$$

Finally, it remains to estimate $J_{4}$. For that, we use Lemma 9 to write

$$
\begin{aligned}
\left|L_{4}\right| & \\
= & \left|T\left(\left(b_{1}(\cdot)-b_{1 i}\right) f \chi_{i},\left(b_{2}(\cdot)-b_{2 j}\right) g \chi_{j}\right)(x)\right| \\
\leq & C 2^{-2 k n}\left\|\left(b_{1}(\cdot)-b_{1 i}\right) f \chi_{i}\right\|_{L^{1}}\left\|\left(b_{2}(\cdot)-b_{2 j}\right) g \chi_{j}\right\|_{L^{1}} \\
\leq & C 2^{-2 k n}\left\|f \chi_{i}\right\|_{L^{p_{1} \cdot(\cdot)}}\left\|\left(b_{1}(\cdot)-b_{1 i}\right) \chi_{i}\right\|_{\left.L^{p_{1}^{\prime} \cdot} \cdot\right)}\left\|g \chi_{j}\right\|_{L^{p_{2}(\cdot)}} \\
& \times\left\|\left(b_{2}(\cdot)-b_{2 j}\right) \chi_{j}\right\|_{L^{p_{2}^{\prime} \cdot(\cdot)}} \\
\leq & C 2^{-2 k n}\left\|f \chi_{i}\right\|_{L^{p^{1} \cdot(\cdot)}}\left\|\left(b_{1}(\cdot)-b_{1 i}\right) \chi_{B_{i}}\right\|_{L^{p_{1}(\cdot)}}\left\|g \chi_{j}\right\|_{L^{p_{2}(\cdot)}} \\
& \times\left\|\left(b_{2}(\cdot)-b_{2 j}\right) \chi_{B_{j}}\right\|_{L^{p_{2}} \cdot(\cdot)}
\end{aligned}
$$


ISRN Mathematical Analysis

7

$$
\begin{aligned}
\leq & C\left\|b_{1}\right\|_{*}\left\|b_{2}\right\|_{*} 2^{-2 k n}\left\|f \chi_{i}\right\|_{L^{p_{1}(\cdot)}}\left\|\chi_{B_{i}}\right\|_{L^{p_{1}^{\prime}(\cdot)}}\left\|g \chi_{j}\right\|_{L^{p_{2}(\cdot)}} \\
& \times\left\|\chi_{B_{j}}\right\|_{L^{p_{2}^{\prime}(\cdot)}} .
\end{aligned}
$$

Thus, by Hölder's inequality and Lemmas 6 and 8, we obtain

$$
\begin{aligned}
& J_{4}=\left\|\left(L_{4}\right) \chi_{k}\right\|_{L^{p(\cdot)}} \\
& \leq C\left\|b_{1}\right\|_{*}\left\|b_{2}\right\|_{*} 2^{-2 k n}\left\|f \chi_{i}\right\|_{L^{p_{1}(\cdot)}}\left\|\chi_{B_{i}}\right\|_{L^{p_{1}(\cdot)}}\left\|g \chi_{j}\right\|_{L^{p_{2}(\cdot)}} \\
& \times\left\|\chi_{B_{j}}\right\|_{L^{p_{2}(\cdot)}}\left\|\chi_{k}\right\|_{L^{p(\cdot)}} \\
& \leq C\left\|b_{1}\right\|_{*}\left\|b_{2}\right\|_{*} 2^{-2 k n}\left\|f \chi_{i}\right\|_{L^{p_{1}(\cdot)}}\left\|\chi_{B_{i}}\right\|_{L^{p_{1}(\cdot)}}\left\|\chi_{B_{k}}\right\|_{L^{p_{1}(\cdot)}} \\
& \times\left\|g \chi_{j}\right\|_{L^{p_{2}(\cdot)}}\left\|\chi_{B_{j}}\right\|_{L^{p_{2}(\cdot)}}\left\|\chi_{B_{k}}\right\|_{L^{p_{2}(\cdot)}} \\
& \leq C\left\|b_{1}\right\|_{*}\left\|b_{2}\right\|_{*}(k-i)\left\|f \chi_{i}\right\|_{L^{p_{1}(\cdot)}} \frac{\left\|\chi_{B_{i}}\right\|_{L^{p_{1}^{\prime}(\cdot)}}}{\left\|\chi_{B_{k}}\right\|_{L^{p_{1}^{\prime}(\cdot)}}} \\
& \times\left\|g \chi_{j}\right\|_{L^{p_{2}(\cdot)}} \frac{\left\|\chi_{B_{j}}\right\|_{L_{2}^{p_{2}^{\prime}(\cdot)}}}{\left\|\chi_{B_{k}}\right\|_{L^{p_{2}^{\prime}(\cdot)}}} \\
& \leq C\left\|b_{1}\right\|_{*}\left\|b_{2}\right\|_{*}\left\|f \chi_{i}\right\|_{L^{p_{1}(\cdot)}} 2^{-(k-i) n \delta_{p_{1}^{\prime}}}\left\|g \chi_{j}\right\|_{L^{p_{2}(\cdot)}} 2^{-(k-j) n \delta_{p_{2}^{\prime}}} \text {. }
\end{aligned}
$$

Combining the estimates for $J_{1}, J_{2}, J_{3}$, and $J_{4}$, we have

$$
\begin{aligned}
\left\|\left[b_{1}, b_{2}, T\right]\left(f \chi_{i}, g \chi_{j}\right) \chi_{k}\right\|_{L^{p(\cdot)}} \leq & C\left\|b_{1}\right\|_{*}\left\|b_{2}\right\|_{*}\left\|f \chi_{i}\right\|_{L^{p_{1}(\cdot)}} \\
& \times(k-i) 2^{-(k-i) n \delta_{p_{1}^{\prime}}}\left\|g \chi_{j}\right\|_{L^{p_{2}(\cdot)}} \\
& \times(k-j) 2^{-(k-j) n \delta_{p_{2}^{\prime}}} .
\end{aligned}
$$

Case II $(i \geq k+2, j \geq k+2)$. We denote $\left(b_{l}\right)_{B_{k}}$ by $b_{l k}$, where $\left(b_{l}\right)_{B_{k}}=\left(1 /\left|B_{k}\right|\right) \int_{B_{k}} b_{l}(x) d x, l=1,2$. In this case, we consider the following decomposition:

$$
\begin{aligned}
& {\left[b_{1}, b_{2}, T\right]\left(f \chi_{i}, g \chi_{j}\right)(x) } \\
&=\left(b_{1}(x)-b_{1 k}\right)\left(b_{2}(x)-b_{2 k}\right) T\left(f \chi_{i}, g \chi_{j}\right)(x) \\
&-\left(b_{1}(x)-b_{1 k}\right) T\left(f \chi_{i},\left(b_{2}(\cdot)-b_{2 k}\right) g \chi_{j}\right)(x) \\
& \quad-\left(b_{2}(x)-b_{2 k}\right) T\left(\left(b_{1}(\cdot)-b_{1 k}\right) f \chi_{i}, g \chi_{j}\right)(x) \\
&+T\left(\left(b_{1}(\cdot)-b_{1 k}\right) f \chi_{i},\left(b_{2}(\cdot)-b_{2 k}\right) g \chi_{j}\right)(x) \\
&= \widetilde{L}_{1}(x)+\widetilde{L}_{2}(x)+\widetilde{L}_{3}(x)+\widetilde{L}_{4}(x) .
\end{aligned}
$$

Thus,

$$
\begin{aligned}
\left\|\left[b_{1}, b_{2}, T\right]\left(f \chi_{i}, g \chi_{j}\right) \chi_{k}\right\|_{L^{p(\cdot)}} & \leq \sum_{m=1}^{4}\left\|\left(\widetilde{L}_{m}\right) \chi_{k}\right\|_{L^{p(\cdot)}} \\
& =\sum_{m=1}^{4} \widetilde{J}_{m} .
\end{aligned}
$$

Let us first compute $\widetilde{J}_{1}$. As in the proof of Theorem 3, in this case we estimate $\widetilde{L}_{1}$ as

$$
\begin{aligned}
\left|\widetilde{L}_{1}\right| \leq & C\left|b_{1}(x)-b_{1 k}\right|\left|b_{2}(x)-b_{2 k}\right| 2^{-i n}\left\|f \chi_{i}\right\|_{L^{1}} 2^{-j n} \\
& \times\left\|g \chi_{j}\right\|_{L^{1}} \\
\leq & C\left|b_{1}(x)-b_{1 k}\right|\left|b_{2}(x)-b_{2 k}\right| 2^{-i n}\left\|f \chi_{i}\right\|_{L^{p_{1}(\cdot)}} \\
& \times\left\|\chi_{i}\right\|_{L^{p_{1}^{\prime}(\cdot)}} 2^{-j n}\left\|g \chi_{j}\right\|_{L^{p_{2}(\cdot)}}\left\|\chi_{j}\right\|_{L^{p_{2}^{\prime}(\cdot)}} .
\end{aligned}
$$

By virtue of generalized Hölder's inequality and Lemmas 6-9, we obtain

$$
\begin{aligned}
& \widetilde{J}_{1}=\left\|\left(\widetilde{L}_{1}\right) \chi_{k}\right\|_{L^{p(\cdot)}} \\
& \leq C 2^{-i n}\left\|f \chi_{i}\right\|_{L^{p_{1}(\cdot)}}\left\|\chi_{B_{i}}\right\|_{L^{p_{1}^{\prime}(\cdot)}} 2^{-j n}\left\|g \chi_{j}\right\|_{L^{p_{2}(\cdot)}}\left\|\chi_{B_{j}}\right\|_{L^{p_{2}^{\prime}(\cdot)}} \\
& \times\left\|\left(b_{1}(x)-b_{1 k}\right)\left(b_{2}(x)-b_{2 k}\right) \chi_{k}\right\|_{L^{p(\cdot)}} \\
& \leq C 2^{-i n}\left\|f \chi_{i}\right\|_{L^{p_{1}(\cdot)}}\left\|\chi_{B_{i}}\right\|_{L^{p_{1}^{\prime}(\cdot)}}\left\|\left(b_{1}(x)-b_{1 k}\right) \chi_{k}\right\|_{L^{p_{1}(\cdot)}} \\
& \times 2^{-j n}\left\|g \chi_{j}\right\|_{L^{p_{2}(\cdot)} \|}\left\|\chi_{B_{j}}\right\|_{L^{p_{2}^{\prime}(\cdot)}}\left\|\left(b_{2}(x)-b_{2 k}\right) \chi_{k}\right\|_{L^{p_{2}(\cdot)}} \\
& \leq C\left\|b_{1}\right\|_{*}\left\|b_{2}\right\|_{*} 2^{-i n}\left\|f \chi_{i}\right\|_{L^{p_{1}(\cdot)}}\left\|\chi_{B_{i}}\right\|_{L^{p_{1}^{\prime}(\cdot)}}\left\|\chi_{B_{k}}\right\|_{L^{p_{1}(\cdot)}} \\
& \times 2^{-j n}\left\|g \chi_{j}\right\|_{L^{p_{2}(\cdot)}}\left\|\chi_{B_{j}}\right\|_{L^{p_{2}^{\prime}(\cdot)}}\left\|\chi_{B_{k}}\right\|_{L^{p_{2}(\cdot)}}
\end{aligned}
$$

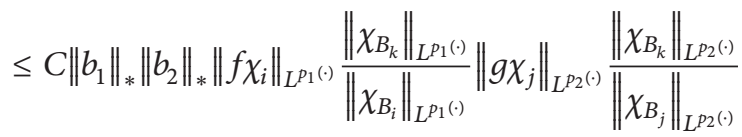

$$
\begin{aligned}
& \leq C\left\|b_{1}\right\|_{*}\left\|b_{2}\right\|_{*}\left\|f \chi_{i}\right\|_{L^{p_{1}(\cdot)}} 2^{(k-i) n \delta_{p_{1}}}\left\|g \chi_{j}\right\|_{L^{p_{2}(\cdot)}} 2^{(k-j) n \delta_{p_{2}}} \text {. }
\end{aligned}
$$

Next, we approximate $\widetilde{J}_{2}$. Using Lemma 9, it is easy to see that

$$
\begin{aligned}
\left|\widetilde{L}_{2}\right| \leq & C 2^{-2 i n}\left|b_{1}(x)-b_{1 k}\right|\left\|f \chi_{i}\right\|_{L^{1}}\left\|\left(b_{2}(\cdot)-b_{2 k}\right) g \chi_{j}\right\|_{L^{1}} \\
\leq & C 2^{-i n}\left|b_{1}(x)-b_{1 k}\right|\left\|f \chi_{i}\right\|_{L^{p_{1}(\cdot)}}\left\|\chi_{i}\right\|_{L^{p_{1}^{\prime}(\cdot)}} \\
& \times 2^{-j n}\left\|g \chi_{j}\right\|_{L^{p_{2}(\cdot)}}\left\|\left(b_{2}(\cdot)-b_{2 k}\right) \chi_{j}\right\|_{L^{p_{2}^{\prime}(\cdot)}} \\
\leq & C\left\|b_{2}\right\|_{*} 2^{-i n}\left|b_{1}(x)-b_{1 k}\right|\left\|f \chi_{i}\right\|_{L^{p_{1}(\cdot)}}\left\|\chi_{B_{i}}\right\|_{L^{\left.p_{1}^{(} \cdot\right)}} \\
& \times(j-k) 2^{-j n}\left\|g \chi_{j}\right\|_{L^{p_{2}(\cdot)}}\left\|\chi_{B_{j}}\right\|_{L^{p_{2}^{\prime}(\cdot)}}
\end{aligned}
$$

Thus, in view of Lemmas 6, 9, and 8, we get

$$
\begin{aligned}
\widetilde{J}_{2}= & \left\|\left(\widetilde{L}_{2}\right) \chi_{k}\right\|_{L^{p(\cdot)}} \\
\leq & C\left\|b_{2}\right\|_{*} 2^{-i n}\left\|f \chi_{i}\right\|_{L^{p_{1}(\cdot)}}\left\|\chi_{B_{i}}\right\|_{L^{p_{1}^{\prime}(\cdot)}}\left\|\left(b_{1}(x)-b_{1 k}\right) \chi_{k}\right\|_{L^{p(\cdot)}} \\
& \times(j-k) 2^{-j n}\left\|g \chi_{j}\right\|_{L^{p_{2}(\cdot)}}\left\|\chi_{B_{j}}\right\|_{L^{p_{2}^{\prime}(\cdot)}}
\end{aligned}
$$


$\leq C\left\|b_{1}\right\|_{*}\left\|b_{2}\right\|_{*} 2^{-i n}\left\|f \chi_{i}\right\|_{\left.L^{p_{1}()}\right)}\left\|\chi_{B_{i}}\right\|_{L^{\left.p_{1}^{(}\right)}}\left\|\chi_{B_{k}}\right\|_{L^{\left.p^{(}\right)}}$ $\times(j-k) 2^{-j n}\left\|g \chi_{j}\right\|_{L^{p^{2} \cdot(\cdot)}}\left\|\chi_{B_{j}}\right\|_{L^{p_{2}^{\prime}(\cdot)}}$

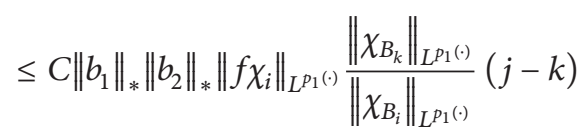
$\times\left\|g \chi_{j}\right\|_{L^{\left.p_{2}()\right)}}\left\|\chi_{B_{k^{\prime}}}\right\|_{L^{p_{2}(\cdot)}}$

$\leq C\left\|b_{1}\right\|_{*}\left\|b_{2}\right\|_{*}\left\|f \chi_{i}\right\|_{L^{p_{1}(\cdot)}} 2^{(k-i) n \delta_{p_{1}}}\left\|g \chi_{j}\right\|_{L^{p_{2} \cdot(\cdot)}}$

$$
\times(j-k) 2^{(k-j) n \delta_{p_{2}}} .
$$

By symmetry, the estimate for $\widetilde{J}_{3}$ is similar to that for $\widetilde{J}_{2}$; thus

$$
\begin{aligned}
\tilde{J}_{3} \leq & C\left\|b_{1}\right\|_{*}\left\|b_{2}\right\|_{*}\left\|f \chi_{i}\right\|_{L^{p_{1}(\cdot)}}(i-k) 2^{(k-i) n \delta_{p_{1}}} \\
& \times\left\|g \chi_{j}\right\|_{L^{p_{2}(\cdot)}} 2^{(k-j) n \delta_{p_{2}}} .
\end{aligned}
$$

Lastly, it remains to compute $\widetilde{J}_{4}$. For this purpose we use generalized Höder's inequality and lemmas 6 and 9 to obtain

$$
\begin{aligned}
& \left|\widetilde{L}_{4}\right|=\left|T\left(\left(b_{1}(\cdot)-b_{1 k}\right) f \chi_{i},\left(b_{2}(\cdot)-b_{2 k}\right) g \chi_{j}\right)(x)\right| \\
& \leq C 2^{-i n}\left\|\left(b_{1}(\cdot)-b_{1 k}\right) f \chi_{i}\right\|_{L^{1}} 2^{-j n} \\
& \times\left\|\left(b_{2}(\cdot)-b_{2 k}\right) g \chi_{j}\right\|_{L^{1}} \\
& \leq C 2^{-i n}\left\|f \chi_{i}\right\|_{L^{p_{1}(\cdot)}}\left\|\left(b_{1}(\cdot)-b_{1 k}\right) \chi_{i}\right\|_{L^{p_{1}^{\prime}(\cdot)}} \\
& \times 2^{-j n}\left\|g \chi_{j}\right\|_{L^{p_{2}(\cdot)}}\left\|\left(b_{2}(\cdot)-b_{2 k}\right) \chi_{j}\right\|_{L^{p^{\prime}(\cdot)}} \\
& \leq C\left\|b_{1}\right\|_{*}\left\|b_{2}\right\|_{*}(i-k) 2^{-i n}\left\|f \chi_{i}\right\|_{L^{p_{1}(\cdot)}}\left\|\chi_{B_{i}}\right\|_{L^{p_{1}^{\prime}(\cdot)}} \\
& \times(j-k) 2^{-j n}\left\|g \chi_{j}\right\|_{L^{p_{2}(\cdot)}}\left\|\chi_{B_{j}}\right\|_{L^{p_{2}^{\prime}(\cdot)}} \\
& \leq C\left\|b_{1}\right\|_{*}\left\|b_{2}\right\|_{*}(i-k) \frac{\left\|f \chi_{i}\right\|_{L^{p_{1}(\cdot)}}}{\left\|\chi_{B_{i}}\right\|_{L^{p_{1}(\cdot)}}}(j-k) \frac{\left\|g \chi_{j}\right\|_{L^{p_{2}(\cdot)}}}{\left\|\chi_{B_{j}}\right\|_{L^{p^{2}(\cdot)}}} \text {. }
\end{aligned}
$$

Thus, an application of Lemma 8 yields

$$
\begin{aligned}
\widetilde{J}_{4}= & \left\|\left(\widetilde{L}_{4}\right) \chi_{k}\right\|_{L^{p(\cdot)}} \leq C\left\|b_{1}\right\|_{*}\left\|b_{2}\right\|_{*}(i-k) \frac{\left\|f \chi_{i}\right\|_{L^{p_{1}(\cdot)}}}{\left\|\chi_{i}\right\|_{L^{p_{1}(\cdot)}}} \\
& \times(j-k) \frac{\left\|g \chi_{j}\right\|_{L^{p_{2}(\cdot)}}\left\|\chi_{k}\right\|_{L^{p(\cdot)}}}{\left\|\chi_{j}\right\|_{L^{p_{2}(\cdot)}}} \\
\leq & C\left\|b_{1}\right\|_{*}\left\|b_{2}\right\|_{*}(i-k)\left\|f \chi_{i}\right\|_{L^{p_{1}(\cdot)}} \frac{\left\|\chi_{B_{k}}\right\|_{L^{p_{1}(\cdot)}}(j-k)}{\left\|\chi_{B_{i}}\right\|_{L^{p_{1}(\cdot)}}}(j) \\
& \times\left\|g \chi_{j}\right\|_{L^{p_{2}(\cdot)}} \frac{\left\|\chi_{B_{k}}\right\|_{L^{p_{2}(\cdot)}}}{\left\|\chi_{B_{j}}\right\|_{L^{p_{2}(\cdot)}}}
\end{aligned}
$$

$$
\begin{aligned}
\leq & C\left\|b_{1}\right\|_{*}\left\|b_{2}\right\|_{*}(i-k)\left\|f \chi_{i}\right\|_{L^{p_{1}(\cdot)}} 2^{(k-i) n \delta_{p_{1}}} \\
& \times(j-k)\left\|g \chi_{j}\right\|_{L^{p_{2}(\cdot)}} 2^{(k-j) n \delta_{p_{2}}} .
\end{aligned}
$$

Combining the estimates for $\widetilde{J}_{1}, \widetilde{J}_{2}, \widetilde{J}_{3}$, and $\widetilde{J}_{4}$, we have

$$
\begin{aligned}
& \left\|\left[b_{1}, b_{2}, T\right]\left(f \chi_{i}, g \chi_{j}\right) \chi_{k}\right\|_{L^{p(\cdot)}} \\
& \leq C\left\|b_{1}\right\|_{*}\left\|b_{2}\right\|_{*}\left\|f \chi_{i}\right\|_{L^{p_{1}(\cdot)}}(i-k) 2^{(k-i) n \delta_{p_{1}}} \\
& \quad \times\left\|g \chi_{j}\right\|_{L^{p_{2}(\cdot)}}(j-k) 2^{(k-j) n \delta_{p_{2}}} .
\end{aligned}
$$

In view of (43), (54), and (64), we arrive at

$$
\begin{aligned}
2^{k \alpha} \| & {\left[b_{1}, b_{2}, T\right]\left(f \chi_{i}, g \chi_{j}\right) \chi_{k} \|_{L^{p(\cdot)}} } \\
\leq & C\left\|b_{1}\right\|_{*}\left\|b_{2}\right\|_{*} E_{1}(k, i) 2^{i \alpha_{1}}\left\|f \chi_{i}\right\|_{L^{p_{1}(\cdot)}} \\
& \times E_{2}(k, j) 2^{j \alpha_{2}}\left\|g \chi_{j}\right\|_{L^{p_{2}(\cdot)}},
\end{aligned}
$$

where for $k, i \in \mathbb{Z}, l=1,2$,

$$
E_{l}(k, i)= \begin{cases}(k-i) 2^{(k-i)\left(\alpha_{l}-n \delta_{p_{l}^{\prime}}\right)}, & \text { if } i \leq k-2, \\ 1, & \text { if } k-1 \leq i \leq k+1, \\ (i-k) 2^{(k-i)\left(\alpha_{l}+n \delta_{p_{l}}\right)}, & \text { if } i \geq k+2\end{cases}
$$

Under the assumption $-n \delta_{p_{l}}<\alpha_{l}<n \delta_{p_{l}^{\prime}}$, it is easy to see that

$$
\sum_{i=-\infty}^{\infty} E_{1}(k, i)^{\gamma}+\sum_{j=-\infty}^{\infty} E_{2}(k, j)^{\gamma}<\infty, \quad \text { for any } \gamma>0 \text {. }
$$

Now by the Minkowski's inequality and (65), we get

$$
\begin{aligned}
& 2^{k \alpha}\left\|\left[b_{1}, b_{2}, T\right](f, g) \chi_{k}\right\|_{L^{p(\cdot)}} \\
& \leq 2^{k \alpha}\left\|\sum_{i=-\infty}^{\infty} \sum_{j=-\infty}^{\infty}\left[b_{1}, b_{2}, T\right]\left(f \chi_{i}, g \chi_{j}\right) \chi_{k}\right\|_{L^{p(\cdot)}} \\
& \leq \sum_{i=-\infty}^{\infty} \sum_{j=-\infty}^{\infty} 2^{k \alpha}\left\|\left[b_{1}, b_{2}, T\right]\left(f \chi_{i}, g \chi_{j}\right) \chi_{k}\right\|_{L^{p(\cdot)}} \\
& \leq C\left\|b_{1}\right\|_{*}\left\|b_{2}\right\|_{*} \sum_{i=-\infty}^{\infty} E_{1}(k, i) 2^{i \alpha_{1}}\left\|f \chi_{i}\right\|_{L^{p_{1}(\cdot)}} \\
& \quad \times \sum_{j=-\infty}^{\infty} E_{2}(k, j) 2^{j \alpha_{2}}\left\|g \chi_{j}\right\|_{L^{p_{2}(\cdot)}} .
\end{aligned}
$$

Finally, by definition and the fact that $1 / q=1 / q_{1}+1 / q_{2}$, we obtain

$$
\begin{aligned}
& \left\|\left[b_{1}, b_{2}, T\right](f, g)\right\|_{\dot{K}_{p(\cdot)}^{\alpha, q}} \\
& =\left\{\sum_{k=-\infty}^{\infty} 2^{k \alpha q}\left\|\left[b_{1}, b_{2}, T\right](f, g) \chi_{k}\right\|_{L^{p(\cdot)}}^{q}\right\}^{1 / q}
\end{aligned}
$$




$$
\begin{aligned}
\leq & C\left\|b_{1}\right\|_{*}\left\|b_{2}\right\|_{*} \\
& \times\left\{\sum_{k=-\infty}^{\infty}\left\{\sum_{i=-\infty}^{\infty} E_{1}(k, i) 2^{i \alpha_{1}}\left\|f \chi_{i}\right\|_{L^{p_{1}}(\cdot)}\right\}^{q_{1}}\right\}^{1 / q_{1}} \\
& \times\left\{\sum_{k=-\infty}^{\infty}\left\{\sum_{j=-\infty}^{\infty} E_{2}(k, j) 2^{j \alpha_{2}}\left\|g \chi_{j}\right\|_{L^{p_{2}(\cdot)}}\right\}^{q_{2}}\right\}^{1 / q_{2}} \\
:= & \left\|b_{1}\right\|_{*}\left\|b_{2}\right\|_{*}\left(\widetilde{I}_{1} \times \widetilde{I}_{2}\right) .
\end{aligned}
$$

It is enough to show that $\widetilde{I}_{1} \leq C\|f\|_{\dot{K}_{p_{1}(\cdot)}^{\alpha_{1}, q_{1}}}$ and $\widetilde{I}_{2} \leq C\|g\|_{\dot{K}_{p_{2}(\cdot)}^{\alpha_{2}, q_{2}}}$. By symmetry, we only give estimates for $\widetilde{I}_{1}$. For $0<q_{1}<\infty$, by inequality $\left(\sum\left|a_{i}\right|\right)^{q_{1}} \leq \sum\left|a_{i}\right|^{q_{1}}$ and inequality (67), we have

$$
\begin{aligned}
\tilde{I}_{1} & \leq C\left\{\sum_{i=-\infty}^{\infty} 2^{i \alpha_{1} q_{1}}\left\|f \chi_{i}\right\|_{L^{p_{1} \cdot(\cdot)}}^{q_{1}} \sum_{k=-\infty}^{\infty} E_{1}(k, i)^{q_{1}}\right\}^{1 / q_{1}} \\
& \leq C\left\{\sum_{i=-\infty}^{\infty} 2^{i \alpha_{1} q_{1}}\left\|f \chi_{i}\right\|_{L^{p_{1} \cdot(\cdot)}}^{q_{1}}\right\}^{1 / q_{1}}=C\|f\|_{\dot{K}_{p_{1}(\cdot)}^{\alpha_{1}, q_{1}}} .
\end{aligned}
$$

For $q_{1}>1$, by Hölder's inequality and inequality (67), we obtain

$$
\begin{aligned}
\widetilde{I}_{1} \leq C\left\{\sum_{k=-\infty}^{\infty} \sum_{i=-\infty}^{\infty} E_{1}(k, i)^{q_{1} / 2} 2^{i \alpha_{1} q_{1}}\left\|f \chi_{i}\right\|_{L^{p_{1}(\cdot)}}^{q_{1}}\right. \\
\left.\times\left\{\sum_{i=-\infty}^{\infty} E_{1}(k, i)^{q_{1}^{\prime} / 2}\right\}^{q_{1} / q_{1}^{\prime}}\right\}^{1 / q_{1}} \\
\leq C\left\{\sum_{i=-\infty}^{\infty} 2^{i \alpha_{1} q_{1}}\left\|f \chi_{i}\right\|_{L^{p_{1}}}^{q_{1}(\cdot)} \sum_{k=-\infty}^{\infty} E_{1}(k, i)^{q_{1} / 2}\right\}^{1 / q_{1}} \\
\leq C\left\{\sum_{i=-\infty}^{\infty} 2^{i \alpha_{1} q_{1}}\left\|f \chi_{i}\right\|_{L^{p_{1}}}^{q_{1} \cdot(\cdot)}\right\}^{1 / q_{1}}=C\|f\|_{\dot{K}_{p_{1}(\cdot)}^{\alpha_{1}, q_{1}}} .
\end{aligned}
$$

Hence, for $0<q_{1}<\infty$

$$
\widetilde{I}_{1} \leq C\|f\|_{\dot{K}_{p_{1}(\cdot)}^{\alpha_{1}, q_{1}}} .
$$

By symmetry, for $0<q_{2}<\infty$, we have

$$
\widetilde{I}_{2} \leq C\|g\|_{\dot{K}_{p_{2}(\cdot)}^{\alpha_{2}, q_{2}}} .
$$

Therefore,

$$
\left\|\left[b_{1}, b_{2}, T\right](f, g)\right\|_{\dot{K}_{p(\cdot)}^{\alpha, q}} \leq C\left\|b_{1}\right\|_{*}\left\|b_{2}\right\|_{*}\|f\|_{\dot{K}_{p_{1}(\cdot)}^{\alpha_{1}, q_{1}}}\|g\|_{\dot{K}_{p_{2}(\cdot)}^{\alpha_{2}, q_{2}}} .
$$

By a similar procedure one can prove that

$$
\begin{aligned}
& \left\|\left(\sum_{h=1}^{\infty}\left|\left[b_{1}, b_{2}, T\right]\left(f_{h}, g_{h}\right)\right|^{r}\right)^{1 / r}\right\|_{\dot{K}_{p(\cdot)}^{\alpha, q}} \\
& \leq\left\|\left(\sum_{h=1}^{\infty}\left|f_{h}\right|^{r_{1}}\right)^{1 / r_{1}}\right\|\left\|_{\dot{K}_{p_{1}(\cdot)}^{\alpha_{1}, q_{1}}}\right\|\left(\sum_{h=1}^{\infty}\left|g_{h}\right|^{r_{2}}\right)^{1 / r_{2}} \|_{\dot{K}_{p_{2}(\cdot)}^{\alpha_{2}, q_{2}}} \\
& \quad \times C\left\|b_{1}\right\|_{*}\left\|b_{2}\right\|_{*}
\end{aligned}
$$

holds for all $f_{h} \in \dot{K}_{p_{1}(\cdot)}^{\alpha_{1}, q_{1}}, g_{h} \in \dot{K}_{p_{2}(\cdot)}^{\alpha_{2}, q_{2}}$, where $1<r_{l}<\infty$ for $l=1,2$ and $1 / r=1 / r_{1}+1 / r_{2}$. Thus, we finish the proof of Theorem 4 .

Remark 11. Note that when $\alpha_{l} \geq n \delta_{p_{l}^{\prime}}$, then the results of Theorems 3 and 4 are no longer true. It needs to replace the variable exponent Herz space $\dot{K}_{p(\cdot)}^{\alpha, q}\left(\mathbb{R}^{n}\right)$ with $H \dot{K}_{p(\cdot)}^{\alpha, q}\left(\mathbb{R}^{n}\right)$ and the Herz-type Hardy spaces with the variable exponent recently introduced in [20]. The boundedness of multilinear singular integral operators from $H \dot{K}_{p(\cdot)}^{\alpha, q}\left(\mathbb{R}^{n}\right)$ to $\dot{K}_{p(\cdot)}^{\alpha, q}\left(\mathbb{R}^{n}\right)$ is still an interesting question that needs to be answered.

Remark 12. Although we considered the 2-linear case, the method can be extended for any $m$-linear case without any essential difficulty.

\section{Conflict of Interests}

The authors declare that there is no conflict of interests regarding the publication of this paper.

\section{References}

[1] R. Coifman and Y. Meyer, "On commutators of singular integrals and bilinear singular integrals," Transactions of the American Mathematical Society, vol. 212, pp. 315-331, 1975.

[2] M. Christ and J. Journé, "Polynomial growth estimates for multilinear singular integral operators," Acta Mathematica, vol. 159, no. 1, pp. 51-80, 1987.

[3] C. Kenig and E. Stein, "Multilinear estimates and fractional integration," Mathematical Research Letters, vol. 6, no. 1, pp. 1$15,1999$.

[4] L. Grafakos and R. H. Torres, "Multilinear Calderón-Zygmund theory," Advances in Mathematics, vol. 165, no. 1, pp. 124-164, 2002.

[5] X. Tao and H. Zhang, "On the boundedness of multilinear operators on weighted herz-Morrey spaces," Taiwanese Journal of Mathematics, vol. 15, no. 4, pp. 1527-1543, 2011.

[6] Q. Wu, "Weighted estimates for multilinear Calderon-Zygmund operators," Advances in Mathematics, vol. 33, pp. 333-342, 2004.

[7] J. Zhou, Y. Cao, and L. Li, "Estimates of commutators for multilinear operators on Herz-type spaces," Applied Mathematics, vol. 25, no. 2, pp. 177-184, 2010.

[8] L. Diening, P. Harjulehto, P. Hästö, and M. Ružička, Lebesgue and Sobolev Spaces with Variable Exponents, vol. 2017 of Lecture Notes in Mathematics, Springer, Heidelberg, Germany, 2011.

[9] S. Samko, "On a progress in the theory of Lebesgue spaces with variable exponent: maximal and singular operators," Integral 
Transforms and Special Functions, vol. 16, no. 5-6, pp. 461-482, 2005.

[10] A. Huang and J. Xu, "Multilinear singular integrals and commutators in variable exponent Lebesgue spaces," Applied Mathematics, vol. 25, no. 1, pp. 69-77, 2010.

[11] M. Izuki, "Herz and amalgam spaces with variable exponent, the Haar wavelets and greediness of the wavelet system," East Journal on Approximations, vol. 15, pp. 87-109, 2009.

[12] M. Izuki, "Boundedness of sublinear operators on Herz spaces with variable exponent and application to wavelet characterization," Analysis Mathematica, vol. 36, no. 1, pp. 33-50, 2010.

[13] A. Almeida and D. Drihem, "Maximal, potential and singular type operators on Herz spaces with variable exponents," Journal of Mathematical Analysis and Applications, vol. 394, no. 2, pp. 781-795, 2012.

[14] S. Samko, "Variable exponent Herz spaces," Mediterranean Journal of Mathematics, vol. 10, no. 4, pp. 2007-2025, 2013.

[15] D. Cruz-Uribe, A. Fiorenza, and C. J. Neugebauer, "The maximal function on variable $L^{p}$ spaces," Annales Academiae Scientiarum Fennicae Mathematica, vol. 28, no. 1, pp. 223-238, 2003.

[16] A. Nekvinda, "Hardy-littlewood maxell operator on $L^{p(x)}\left(\mathbb{R}^{n}\right)$," Mathematical Inequalities and Applications, vol. 7, no. 2, pp. 255265, 2004.

[17] D. Cruz-Uribe, A. Fiorenza, J. M. Martell, and C. Pérez, "The boundedness of classical operators on variable $L^{p}$ spaces," Annales Academiae Scientiarum Fennicae Mathematica, vol. 31, no. 1, pp. 239-264, 2006.

[18] O. Kovóčik and J. Rakosnk, "On spaces $L^{p(x)}$ and $W^{k, p(x), "}$ Czechoslovak Mathematical Journal, vol. 41, pp. 592-618, 1991.

[19] M. Izuki, "Boundedness of commutators on Herz spaces with variable exponent," Rendiconti del Circolo Matematico di Palermo, vol. 59, no. 2, pp. 199-213, 2010.

[20] H. Wang and Z. Liu, "The Herz-type Hardy spaces with variable exponent and their Applications," Taiwanese Journal of Mathematics, vol. 16, no. 4, pp. 1363-1389, 2012. 


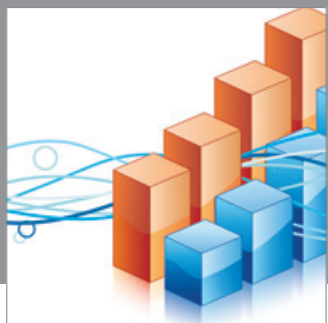

Advances in

Operations Research

mansans

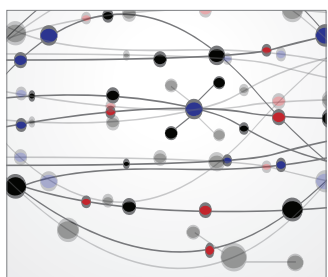

The Scientific World Journal
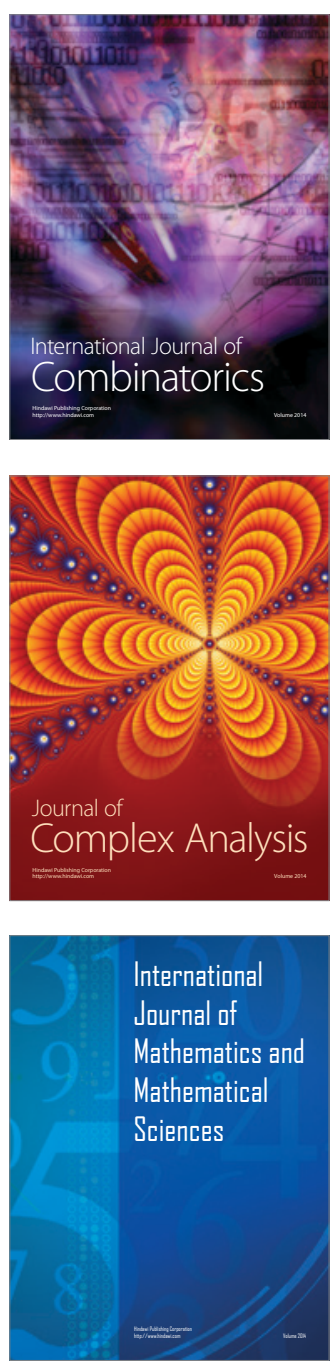
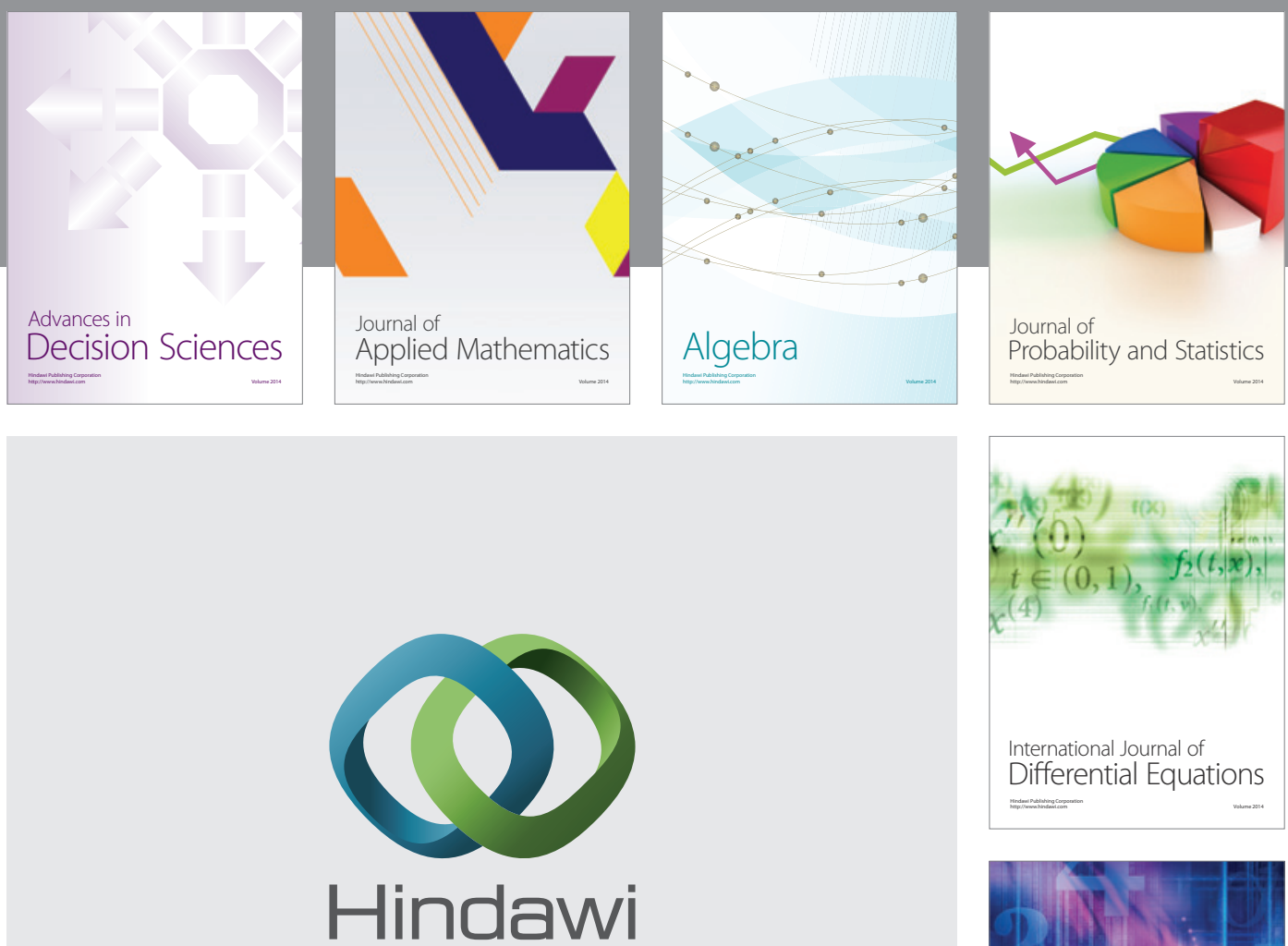

Submit your manuscripts at http://www.hindawi.com
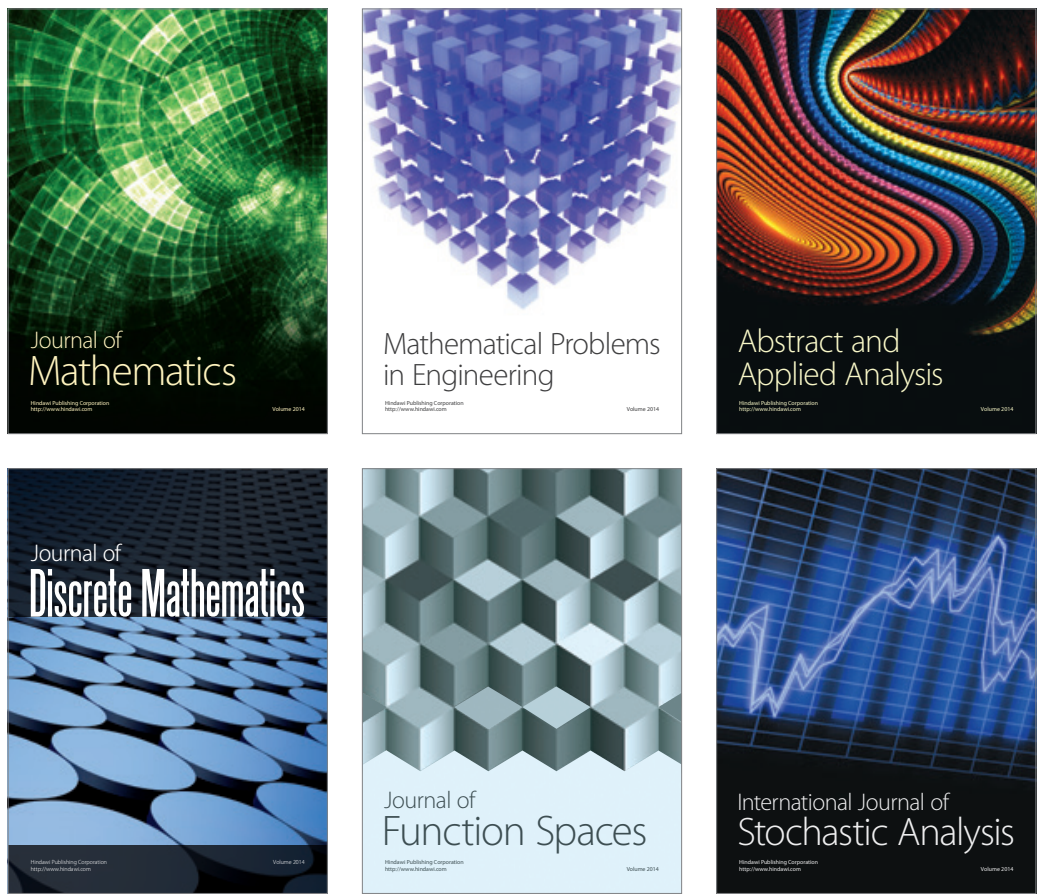

Journal of

Function Spaces

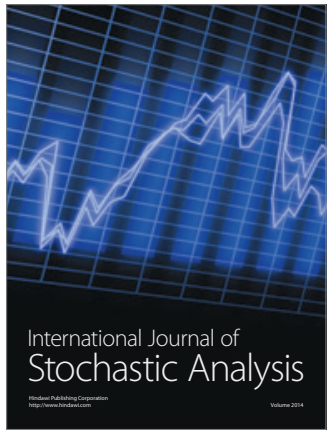

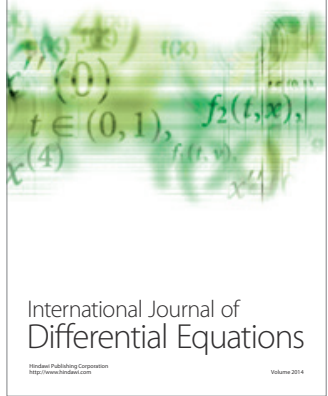
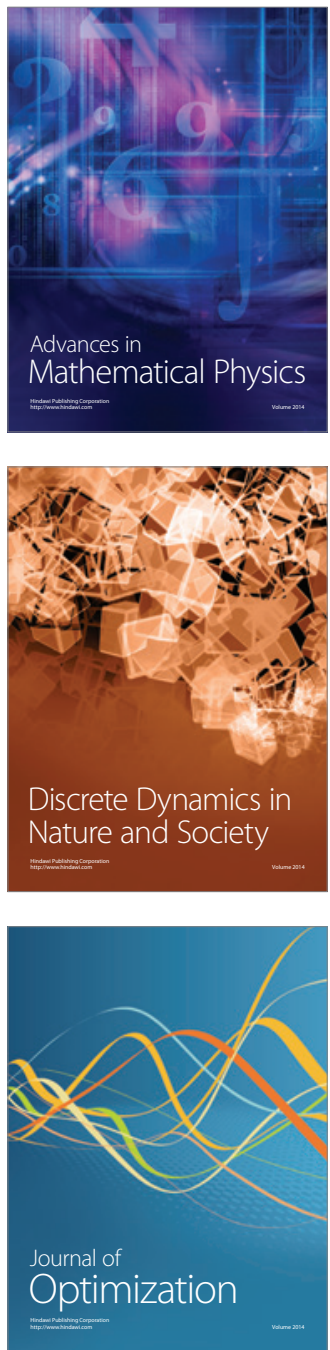